the concentration of mast cells is increased in the synovium of affected joints. Mast cells significantly influence angiogenesis through the production of proangiogenic cytokines, including ANGPTL4.

Conclusion: Changes of the level of ANGPTL4 in the serum of patients with RA may be a potential biomarker of disease activity, severity of neovascularization, inflammation and development of bone erosion.

Disclosure of Interests: None declared

DOI: 10.1136/annrheumdis-2019-eular.7868

\section{AB1119 THE PRESENCE OF SYNOVITIS IS THE MAIN FACTOR INFLUENCING THE DEVELOPMENT OF PAIN SYNDROME IN ARTHRITIS OF THE KNEE JOINT}

Ninel Aleksandrova ${ }^{1}$, Natalia Nikitina ${ }^{1}$, Vladislav Aleksandrov ${ }^{1,2}$, Ludmila Shilova $^{2}$, Andrey Aleksandrov ${ }^{1,2} .{ }^{1}$ Research Institute of Clinical and Experimental Rheumatology named after A.B. Zborovsky, Volgograd, Russian Federation; ${ }^{2}$ Volgograd State Medical University, Hospital Therapy, Volgograd, Russian Federation

Background: Dysfunctions and pain syndrome in lesions of the knee joint can significantly discomfort a sick person and lead to a persistent decrease in physical activity and disability. Often pain syndrome precedes radiographic appearance of the structural changes in the joint and is accompanied by an increase in number of different changes in the synovium according to ultrasound investigation.

Objectives: To investigate the clinical significance of ultrasound criteria of changes in the synovial membrane of the knee joint cavity and its role in the assessment of pain in gonarthrosis.

Methods: 30 people aged 30 to 50 years with osteoarthritis of the knee joint were under observation; assessment of the severity of pain in the knee when walking was at least $40 \mathrm{~mm}$ on a visual analogue scale (VAS). Ultrasound examination of the knee joint was carried out according to standard procedure using a linear sensor with a frequency of $05.12 \mathrm{MHz}$ in an ultrasonic diagnosis system Accuvix V10 (Samsung Medison, Korea).

Results: The evaluation of ultrasound changes was performed in the upper inversion of the knee joint according to the following criteria: the severity of intraarticular effusion (1), synovial proliferation (2), local vascularization of the synovial membrane by power doppler (3). All patients were divided into three groups, according to the severity of pain in the knee joint: group I (12 people) - 41-59 mm, group II (10 people) - 60$79 \mathrm{~mm}$, group III (8 people) - 80-100 mm on the VAS scale. By comparing changes in the knee joint by ultrasound data in patients of different groups, the following results were obtained: group I: severity of intraarticular effusion - 10 people (minimal changes in $60 \%$, moderate in $20 \%$, expressed in $20 \%$ ), synovial proliferation - 4 people (moderate changes in $50 \%$, expressed in $50 \%$ ), local vascularization of synovium 6 people (minimal changes in $66.7 \%$, moderate in $16.7 \%$, expressed in $16.6 \%)$; Group II: severity of intraarticular effusion - 9 people $(55.6 \%$, $22.2 \%$ and $22.2 \%)$, synovial proliferation - 3 people $(0 \%, 33.3 \%$ and $66.7 \%$,), local vascularization of the synovial membrane - 4 people $(25 \%$, $25 \%$ and $50 \%$, respectively); group III: severity of intraarticular effusion 8 people $(62.5 \%, 12.5 \%$ and $25 \%)$, synovial proliferation - 5 people $(20 \%, 40 \%$ and $40 \%)$, local vascularization of the synovial membrane - 3 people (per $33.3 \%$, respectively).

Conclusion: The use of ultrasound in the diagnosis of diseases of the knee joints allows to reliably determine the structural and functional changes in all components of the knee joint. The severity of pain in gonarthrosis is most associated with the presence of synovitis in the joint.

Disclosure of Interests: None declared

DOI: 10.1136/annrheumdis-2019-eular.7065

\section{AB1120 DIAGNOSTIC ROLE OF NEUROMUSCULAR ULTRASOUND IN CUBITAL TUNNEL SYNDROME}

Fatma Ali ${ }^{1}$, Mona Nasr ${ }^{1}$, Ahmed Hafez ${ }^{2}$, Adham Khalil ${ }^{3} .{ }^{1}$ Minia university, Reumatology and Rehabilitation, Minia, Egypt, ${ }^{1}$ Minia university, Reumatology and Rehabilitation, Minia, Egypt, ${ }^{3}$ New Kasr El Eini Teatching Hospital, Cairo University, Cairo, Egypt

Background: Cubital tunnel syndrome (CUTS) is the second most common compressive neuropathy of the upper limb following carpal tunnel syndrome and is the most common site for entrapment for the ulnar nerve
Objectives: Our aim is to evaluate the role of ultrasonography (US) as a diagnostic tool for Cubital tunnel syndrome (CuTS) in comparison with nerve conduction study (NCS).

Methods: twenty elbows with CuTS and twenty asymptomatic controls were assessed by NCS and underwent ultrasonography of elbows. Data from patients and controls were compared to determine the diagnostic relations in patients with CUTS and the grade of severity

Results: There was a high degree of correlation between NCS of the ulnar nerve, clinical parameters and variable US measurements. The CSA of the ulnar nerve was the most sensitive parameter and a cut-off point of $9.5 \mathrm{~mm}^{2}$ behind medial epicondyle was found to be $100 \%$ sensitive and $80 \%$ specific. The ulnar nerve ratios (UNR) had a diagnostic accuracy of $95 \%$ with $85 \%$ specificity.

Conclusion: Ultrasonographic measurements of the ulnar nerve CSA and UNR have a comparable diagnostic value as a non-invasive and an alternative modality for the evaluation of CuTS

\section{REFERENCES}

[1] Simon N, Ralph J, Poncelet A, Engstrom J, Chin C, Kliot M. A comparison of ultrasonographic and electrophysiologic 'inching'in ulnar neuropathy at the elbow. Clin Neurophysiol. 2015;126(2):391-398.

[2] Omejec G, Podnar S. Normative values for short segment nerve conduction studies and ultrasonography of the ulnar nerve at the elbow. Muscle nerve. 2015;51(3):370-377.

[3] Yoon J, Walker F, Cartwright M. Ultrasonographic swelling ratio in the diagnosis of ulnar neuropathy at the elbow. Muscle nerve. 2008;38 (4):1231-1235

[4] Bayrak A, Bayrak I, Turker H, Elmali M, Nural M. Ultrasonography in patients with ulnar neuropathy at the elbow: Comparison of cross sectional area and swelling ratio with electrophysiological severity. Muscle nerve. 2010;41(5):661-666.

Disclosure of Interests: None declared

DOI: 10.1136/annrheumdis-2019-eular.4629

\section{AB1121 EFFICACY AND SAFETY OF ULTRASOUND GUIDED ASPIRATION AND INTRA LESIONAL CORTICOSTEROIDS INJECTION OF RUPTURED BAKER'SCYST}

Mohammed A. Mortada ${ }^{1}$, Youmna A. Amer ${ }^{2} .{ }^{1}$ Faculty of medicine,Zagazig University, Reheumatology and Rehabilitation, Zagazig, Egypt, ${ }^{2}$ Faculty of medicine zagazig university, Rheumatology and rehabilitation, Zagazig, Egypt

Background: Baker's cyst is the most common mass in the popliteal fossa and results from fluid distension of the gastrocnemio-semimembranosus bursa. The most common complication of Baker's cyst is the rupture or dissection of fluid into the adjacent proximal gastrocnemius muscle belly, which results in a pseudothrombophlebitis syndrome mimicking symptoms of DVT.

Treatment of ruptured Baker cysts ranged from conservative management to surgical resection.

Ultrasonographic guided aspiration and corticosteroids injection may be an effective and easy method of management of these cases.

Up to the best of our knowledge, this is the first study to detect the efficacy and safety of ultrasonographic guided aspiration and injections of ruptured Baker cysts.

Objectives: To evaluate the efficacy and safety of ultrasonographic guided aspiration and local corticosteroids of ruptured Baker cysts based on follow-up clinical and sonographic results.

Methods: A retrospective study was conducted on 42 patients (12 males and 30 females, mean age $36+/-10$ SD years) affected by a ruptured Baker cysts associated to knee joint disorders in the period between January 2013 to January 2019. The diagnosis was done by clinical presentation of acute calf pain, swelling, tenderness at the calf muscles and ultrasonographic evidences of ruptured backer cysts in the form of free fluid collection in the calf connected to a well defined cyst at the back of knee.

All cases were treated by ultrasonographic guided aspiration and intra lesional injection of corticosteroids once or twice one week a part. Follow up were done on a weekly basis until complete resolution of symptoms then 3 months later. Visual analogue scale (VAS) for calf pain and Rauschning-Lindgren and Lysholm Knee Scoring Scales were used to assess pre/post-injection knee functions.

Results: The primary diagnoses to patients presented with ruptured Baker cyst in this study were as follow: 18 (42.8\%) cases with rheumatoid 